\title{
A Multi Psychology Accounts CSD Model for ISC Bi-Level Distribution Network
}

\author{
Quan $\mathrm{Lu}^{1}$, Jing Chen ${ }^{2}$ and Junping Qiu ${ }^{1}$ \\ 1 Research Center for China Science Evaluation, Wuhan University, \\ Wuhan, 430072, P.R.China \\ mrluquan@sina.com \\ 2 Department Of Computer Science, Central China Normal University, \\ Wuhan, 430079, P.R.China
}

\begin{abstract}
The researches on Supply Chain are mainly about the conjunction mechanism and efficiency in Integrated Supply Chain to enhance its competitiveness and the cooperation between its members. But most of those belong to the standard finance field, and few about the factors of human mental or psychology. We studied the satisfaction degree models of customer with mental account based on the customer satisfaction degree (CSD) model and found they are too simplified, not reasonable and inaccurate. This paper suggests a CSD model based on multi artificial psychology accounts, which quantify exact customer psychology using cognition and artificial psychology methods, and then describes an ISC bi-level distribution network model based on this CSD model.
\end{abstract}

\section{Introduction}

A typical supply chain consists of three fundamental stages: material procurement, production and distribution. Procurement and production are highlighted under traditional manufacture environment. Since the early $1980 \mathrm{~s}$, the focus of supply chain management has been moving towards the demand side [1-3], and supply chains are becoming "demand chains" driven by the market and customers. The researches on Supply Chain are mainly about the conjunction mechanism and efficiency in Integrated Supply Chain to enhance its competitiveness and the cooperation between its members since year $2000[4,5]$, typically the researches on bi-level distribution network. But most of those belong to the standard finance field, and few about the factors of human psychology. We studied the satisfaction degree models of customer $[6,7]$ with mental account based on the CSD model and found they are too simplified, not reasonable and inaccurate. For example, simple mental classification in Multiple Mental Account researches of a consumer like "conservative" and "risky" is obviously not enough for a clothes supply chain system, and more accurate and

Please use the following format when citing this chapter:

Lu, Q., Chen, J., Qiu, J., 2007, in IFIP International Fedcration for Information Processing, Volume 251, Integration and Innovation Orient to L-Society Volume1, Wang, W. (Idds), (Boston: Springer), pp. 417-422. 
effective method are needed to satisfy the customer. Our project aims to improve the customer satisfaction level/degree and increase the enterprise's competitive power through cognitive and artificial psychology technologies.

This paper analyzes the multi psychology accounts structure of consumers in section 2. Section 3 presents a customer satisfaction degree model based on artificial psychology, which quantify exact customer psychology using cognition and artificial psychology methods. Section 4 describes a chance constrained programming model of the ISC bi-level distribution network based on artificial psychology. The conclusions are stated in section 5 .

\section{The Multi Psychology Accounts of Consumers}

The optimization problem of the ISC bi-level distribution network has gone beyond the field of traditional standard finance. Behavioral Portfolio theory [8] and Affective Computing theory will help to its solving, from which we describe bi-level distribution network design problem [9] under distributed multimanufacturer, multi-distributor and multi psychology accounts of multiconsumer scenarios as figure 1. The decision psychology of a user is a hierarchical structure. So the irrational optimization problem of the ISC bi-level distribution network can be translated into one of optimizing customer demands with multi psychology accounts.

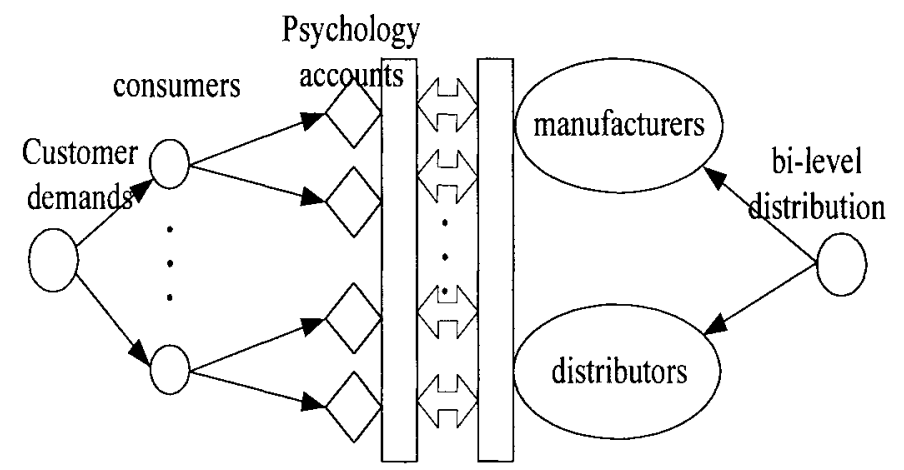

Fig. 8. An ISC bi-level distribution network model with multi psychology accounts of consumer

\section{A CSD Model Based on Multi Artificial Psychology Accounts}

\subsection{Analysis of Customer Psychology Accounts}

To model customer's multi psychology accounts, the first thing we should do is to find how many accounts there are. This can be got from history transaction files through data preparation and clustering $[10,11]$.

Each user session in a user session file can be thought of in two ways; either as a single transaction of many page references, or a set of many transactions 
each consisting of a single page reference. The goal of transaction identification is to dynamically create meaningful clusters of references for each user.

Clustering algorithms are effective for this situation. We consider using a multivariate k-means algorithm [10] which calls k-means algorithm time and again to obtain transaction clusters.

$\mathrm{K}$-means is one of the simplest unsupervised learning algorithms that solve the well known clustering problem. The procedure follows a simple and easy way to classify a given data set through a certain number of clusters (assume $k$ clusters) fixed a priori. The main idea is to define $k$ centroids, one for each cluster. These centroids shoud be placed in a cunning way because of different location causes different result. So, the better choice is to place them as much as possible far away from each other. The next step is to take each point belonging to a given data set and associate it to the nearest centroid. When no point is pending, the first step is completed and an early groupage is done. At this point we need to re-calculate $k$ new centroids as barycenters of the clusters resulting from the previous step. After we have these k new centroids, a new binding has to be done between the same data set points and the nearest new centroid. A loop has been generated. As a result of this loop we may notice that the $\mathrm{k}$ centroids change their location step by step until no more changes are done. In other words centroids do not move any more.

\subsection{Modeling of Customer Artificial Psychology}

To illustrate the modeling of customer artificial psychology clearly, we take one cluster to consider first. Based on Cooley's user model [11] the user query and answer in user transaction file and user cognitive queries files is enhanced with cognitive psychology and the subjective expected utility is assigned. Considering the utilities for different combinations of preferences within a query a trade-off between query complexity or processing costs and an expected improvement of the query result has to be optimized. This query is processed by the database retrieval system and the results are returned to the user.

The modeling work is based on Quantification Theory I [10] and factor analysis theory. Firstly, there is a user preference value correspondence with the adjacent pair, and we call them average of the representative pairs. We can get the average value through questionnaire.

$$
Y=\left[\begin{array}{llll}
y_{1} & y_{2} & \cdots & y_{n}
\end{array}\right]^{T}
$$

Then we can measure the characters of the items or products like value $(0-$ $100)$ of cold or warm. The corresponding reactor matrix is:

$$
\begin{aligned}
& X=[\delta(j, k)]= \\
& \left(\begin{array}{cccccccccc}
\delta(11) & \ldots & \delta\left(1 r_{1}\right) & \delta(21) & \ldots & \delta\left(2 r_{2}\right) & \ldots & \delta(m 1) & \ldots & \delta\left(m r_{m}\right) \\
\delta_{2}(11) & \ldots & \delta_{2}\left(1 r_{1}\right) & \delta_{2}(21) & \ldots & \delta_{2}\left(2 r_{2}\right) & \ldots & \delta_{2}(m 1) & \ldots & \delta_{2}\left(m r_{m}\right) \\
. . & . . & . . & . . & . . & . . & . . & . . & . . & . . \\
. . & . . & . . & . . & . . & . . & . . & . . & . . & . . \\
\delta_{n}(11) & \ldots & \delta_{n}\left(1, r_{1}\right) & \delta_{n}(21) & \ldots & \delta_{n}\left(2 r_{2}\right) & \ldots & \delta_{n}(m 1) & \ldots & \delta_{n}\left(m r_{m}\right)
\end{array}\right)
\end{aligned}
$$

Then each case can be written in the form of reactor matrix. According to Quantification Theory I : 


$$
b=\left(X^{\prime} X\right)^{-1} X^{\prime} Y
$$

Now we obtain the quantification relationship of the adjective pairs and the case, with $\mathrm{Y}$ is a column vector in formula 1 and its value is the average of the previously evaluation corresponding to an adjective pair. Here $\mathrm{X}$ is the reactor matrix in formula 2. All values of $b$ correspond with the adjective pairs can be obtained. By replacing $b$ with its expression, we can calculate the evaluation $Y$ of case with the help of the predictive formula

$$
\hat{Y}=X b
$$

Subject to:

$$
r_{\hat{y} y}=\sqrt{\frac{\sum_{i=1}^{n}\left(\hat{y}_{i}-\bar{y}\right)^{2}}{\sum_{i=1}^{n}\left(y_{i}-\bar{y}\right)^{2}}} \geq 0.85
$$

$\hat{Y}$ is the predicted value of a certain customer's satisfaction degree to a certain product in a certain psychology account.

\subsection{A CSD Model Based on Multi Artificial Psychology and Utility Theory}

Customer artificial psychology built in section 3.2 can not be used directly in ISC bi-level distribution network design. With the above foundation we propose a customer satisfaction degree model based on artificial psychology and utility theory. It can be described as following:

The customer satisfaction degree of customer $i$ in psychology account $p$ relative to account $r$ as following:

$$
S_{i p r}=\sum_{k=1}^{n} a_{i k} \cdot u\left(x_{i p k}-x_{i r k}\right)
$$

Where $x_{i j k}$ is the satisfaction value of customer $i$ 's psychology account $j$ to character $k$ of the product, $a_{i k}$ is satisfaction degree coefficient: $0 \leq a_{i k} \leq 1$, 且 $\sum_{k=1}^{n} a_{i k}=1$ and $u(x)$ is the utility function. $u(x) \geq 0$ as $x \geq 0$ and $u(x) \leq 0$ as $x \leq 0$. And $x_{i j}$ is the predicted customer satisfaction $\hat{Y}$ in formula 4 .

\section{A Psychology-based Chance Constrained Programming Mode of ISC Bi-level Distribution Network}

The following notation is used in the model for ISC bi-level distribution network design. 
$x_{k l}=$ the amount shipped from manufacturer $k$ to distribution center $l$.

$y_{l l}=$ binary variables, denote whether it is a distribution center.

$y_{l r}=$ binary variables, denote whether ship products from distribution center $l$ to distributor $r$.

$d_{r}=$ random variables, the demand amount of customer region $r$.

$f_{k}=$ random variables, the production capacity of manufacturer $k$.

\section{Constants}

$a_{k l}=$ unit cost of shipping from manufacturer $k$ to distribution center $l$.

$b_{l}=$ fixed cost of constructing distribution center $l$.

$M_{l}=$ fixed cost of constructing distributor $l$.

$e_{l r}=$ unit cost of shipping from distribution center $l$ to distributor $r$.

Then we can convert the modeling problem of the ISC bi-level distribution network with multi psychology accounts into one of expected value goal programming with chance constrains according to Zhao Xiaoyu's researches [12].

\section{Model}

$$
\left.\min \left\{\sum_{k=1}^{K L} \sum_{l=1}^{L} a_{k l} x_{k l}+\sum_{l=1}^{N} b_{l} y_{u}+M_{l}\left(1-y_{u}\right)\right]+\sum_{k=1}^{L} \sum_{l=1}^{N} e_{l r} d_{r} y_{l r}+\xi\right\}
$$

\section{Subject to:}

$$
\begin{aligned}
& \xi=\sum_{i=1}^{l}\left(k_{i} \sum_{j=1}^{n} P_{i j} S_{i j} W_{(j)}\right) \\
& \sum_{l=1}^{L} x_{k l} \leq f_{k}, k=1,2, \cdots, K ; \\
& \sum_{l=1}^{N} y_{u}=L, \sum_{l-1}^{L} y_{l r}=1, r=1,2, \cdots, K ; \\
& \sum_{r=1, r \neq l}^{N} y_{l r} \leq(N-1) y_{l l}, l=1,2, \cdots, N ; \\
& \sum_{r=1}^{N} d_{r} y_{l r} \leq \sum_{k=1}^{N} x_{k l}, j=1,2, \cdots, L ; \\
& x_{k l} \geq 0, y_{l r} \in(0,1), \forall k, l, r_{\circ}
\end{aligned}
$$

\section{Conclusions}

Customer satisfaction degree (CSD) model in ISC bi-level distribution network design problem considering customer psychology is investigated in this paper. It describes the hierarchical structure of customer psychology, presents the method 
to model customer artificial psychology, proposes a customer satisfaction degree model based on multi artificial psychology accounts, and then converts the optimization problem of the ISC bi-level distribution network with multi psychology accounts into one of expected value goal programming model with chance constrains, and the implementing and optimization in a real ISC bi-level distribution network of this model will be our future works.

This model covers the following features by considering customer's multi psychologies: more consistency with customer, higher customer satisfaction degree, reasonable optimization and adaptability to demanding waves under bounded rationality of customers.

\section{Acknowledgements}

This research was supported by the National Natural Science Foundation of China under Grant No. 70673071.

\section{References}

1. T. Davis, Effective supply chain management, Sloan Management Review, Summer, 34(4), 5-46 (1993).

2. J.T. Douglas and M.G. Paul, Coordinated supply chain management, European Journal of Operational Research, 94(1), 1-15(1996).

3. S. Bylka, A dynamic model for the single-vendor, multi-buyer problem, International Journal of Production Economics, 59(3), 297-304(1999).

4. P. H. Zipkin, Supply chain management: reflections, interpretations and predictions, Global SCM Conference, Berlin: Springer-Verlage Press (2002) .

5. F. Cheng, M. Ettl and G. Lin, Inventory-service optimization in configureto-order systems, Manufacturing and Service Operations Managemen,t 4(2), 114-132(2002).

6. M. Friedman and L.J. Savage, The utility analysis of choices involving risk, Journal of Political Economy, 56(4), 279-304(1948).

7. Y.K. Ma and X.W. Tang, Decision-making methods for behavioral portfolio choice, Journal of systems engineering, 18 (1), 71-76(2003).

8. S. Hersh and S. Meir, Behavioral Portfolio Theory, The Journal of Financial and Quantitative Analysis, 35( 2), 127-151 (2000).

9. J.J. Jiang etc. Optimization of the ISC Bi-Level Distribution Network with Multiple Mental Accounts, Industrial Engineering Journal, 8 (3), 12-17(2005).

10. Q. Lu, J. Chen and B. Meng, Web Personalization based on Artificial Psychology, WISE Workshop on Web Information Access and Digital Library, LNCS4256, 223-229(2006).

11. R. Cooley, B. Mobasher and J. Srivastava, Data preparation for mining World Wide Web browsing patterns, Journal of Knowledge and Information Systems, 1(1) (1999).

12. X.y.Zhao etc, An Optimization Model for Distribution Network Design with Uncertain Customer Demands and Production Capacity,0-7803-8971-9/05 IEEE (2005). 